\title{
Desenvolvimento de gelatina funcional de erva-mate
}

\author{
Development of functional yerba-mate jelly
}

\section{Kleber Alves Santos Berté ${ }^{\text {* }}$ Dayane Rosalyn Izidoro ${ }^{\mathrm{I}}$ Fabiana Lemos Goularte Dutra ${ }^{\mathrm{II}}$ Rosemary Hoffmann-Ribani ${ }^{I}$}

\section{RESUMO}

\begin{abstract}
O objetivo deste estudo foi desenvolver formulações para gelatina funcional com extrato de erva-mate verde $e$ fibras solúveis como inulina (INU), frutooligossacarídeos (FOS) $e$ a polidextrose (PD), avaliando o efeito desses ingredientes na gelatina funcional por meio da análise física da textura (firmeza, consistência e coesividade), análise sensorial (sabor e preferência de compra) e composição química. As formulações INU, PD e composição INU/PD/FOS apresentaram textura desejável para uma sobremesa de gelatina, não diferindo $(P>0,05)$ do padrão com sacarose. Pela análise sensorial, a gelatina funcional com INU obteve o maior índice hedônico para sabor e preferência de compra acima de 70\%, superior ao padrão. Considerando os resultados obtidos neste estudo, a aplicação tecnológica do extrato de erva-mate verde e das fibras solúveis apresenta evidente potencial para o desenvolvimento de alimentos saudáveis $e$ funcionais.
\end{abstract}

Palavras-chave: alimentos funcionais, Ilex paraguariensis, inulina.

\section{ABSTRACT}

The objective of this study was to develop formulations for functional jelly with extract of green yerbamate and soluble fibers as inulin (INU), fructooligosaccharides $(F O S)$ and polidextrose (PD), evaluating the effect of those ingredients in the functional jelly through texture physical analysis (firmness, consistence, cohesiveness), sensorial analysis (flavor and purchase preference) and chemical composition. The formulations INU, PD and composition INU/PD/FOS presented desirable texture for jelly dessert and not differing $(P>0.05)$ of the reference standard with sucrose. For the sensorial analysis the functional jelly with INU obtained the largest index hedonic for flavor and purchase preference above $70 \%$, higher than the reference standard. Considering the results obtained in this study, the technological application of green yerba-mate extract and of soluble fibers, presents evident potential for the development of healthy and functional foods.

Key words: functional foods, ilex paraguariensis, inulin.

\section{INTRODUÇÃO}

Os alimentos funcionais são aqueles alimentos ou ingredientes que, além das funções nutricionais básicas, apresentam efeitos benéficos para a saúde, quando consumidos como parte da dieta habitual. Os alimentos funcionais são capazes de reduzir o risco de algumas doenças e auxiliar em funções fisiológicas do organismo (STARK \& MADAR, 1994). Os principais alimentos funcionais são: fibras (psyllium, polidextrose e goma guar), ácidos graxos poliinsaturados (ômega 3,6), substâncias bioativas de plantas (polifenóis, cafeína e carotenóides), fibras prebióticas (inulina e frutooligossacarídeos) e os microorganismos probióticos (WAITZBERG, 2000).

A erva-mate (Ilex paraguariensis A.St.-Hil) é uma árvore nativa da América do Sul e é considerada um produto de origem florestal não madeirável. Suas

'Programa de Pós-graduação em Tecnologia de Alimentos (PPGTA), Universidade Federal do Paraná (UFPR), CP 19011, 81531 990, Curitiba, PR, Brasil.

IPrograma de Pós-graduação em Ciência e Tecnologia Agroindustrial, Universidade Federal de Pelotas(UFPel), Pelotas, RS, Brasil. E-mail: kleber.berte@gmail.com. Autor para correspondência. 
folhas apresentam diferentes compostos bioativos que contribuem para ampliar o seu uso e aplicação industrial em diversos produtos, além do tradicional chimarrão. As substâncias bioativas que têm despertado o interesse em pesquisas com a erva-mate são os compostos fenólicos e a cafeína (PARANÁ, 2000). Os principais compostos fenólicos encontrados na ervamate são o ácido cafeíco, a rutina e os derivados dos ácidos clorogênicos com propriedades antioxidantes (FILIP et al., 2000). Esses polifenóis apresentam efeitos sobre a absorção intestinal da glicose, resultando em um menor índice glicêmico do esperado na dieta (CLIFFORD, 2004).

As fibras solúveis são carboidratos complexos como a inulina (INU), os frutooligossacarídeos (FOS) e a polidextrose (PD). A inulina e os frutooligossacarideos são polissacarídeos de reserva extraídos da raiz da chicória (Cichorium intybus) e apresentam propriedades tecnológicas distintas. Essas fibras prebióticas são seguras para diabéticos, estimulam o crescimento seletivo de bactérias intestinais promotoras de saúde, especialmente as bifidobactérias, que contribuem para melhor absorção de nutrientes (CARABIN \& FLAMM, 1999). A polidextrose é um polímero da condensação da dextrose altamente ramificado e apresenta os benefícios fisiológicos das fibras, tais como aumento da velocidade do trânsito intestinal e a atenuação da absorção da glicose (FLOOD et al., 2004).

A sobremesa de gelatina é um produto de sabor agradável elaborado pela mistura de água, gelatina, sacarose e aditivos, podendo apresentar alegação funcional e/ou de saúde, pela adição de fibras ou ingredientes bioativos (fonte de polifenóis) (BRASIL, 2002) ou, ainda, pode ser elaborada para diabéticos, com a substituição dos açucares por fibras e/ou edulcorantes (BRASIL, 1998a).

A crescente preocupação com uma alimentação saudável que, além de nutrir, promova a saúde coloca alguns alimentos e ingredientes funcionais na lista de preferência de um número cada vez maior de consumidores. $\mathrm{O}$ desenvolvimento de novos alimentos funcionais e para fins especiais contribui para a inserção das indústrias nesse mercado. Dessa forma, o presente trabalho teve como objetivo o desenvolvimento de formulações para o preparo de gelatina funcional com extrato de erva-mate verde e fibras solúveis, além de analisar a textura, a composição química e realizar a análise sensorial para verificar o sabor e a preferência de compra das formulações elaboradas, em comparação a gelatina padrão.

\section{MATERIAL E MÉTODOS}

A erva-mate (Ilex paraguariensis $\mathrm{St}-\mathrm{Hil}$ ), proveniente do municipio de São Mateus do Sul-PR, foi cedida pela empresa Baldo S/A. As folhas da ervamate foram submetidas ao processamento industrial padrão de secagem e moagem para obter a erva-mate em pó tipo chimarrão. Para obtenção do extrato aquoso, $1 \mathrm{~kg}$ de erva-mate pó foi acrescido de $10 \mathrm{~L}$ de água potável e mantido em decocção durante 30 minutos à temperatura de $85^{\circ} \mathrm{C}$ (BURGARDT, 2000). O extrato aquoso foi submetido à secagem em spray dryer para obter o extrato de erva-mate em pó com maior concentração de compostos bioativos em relação às folhas. Os demais ingredientes foram adquiridos de fornecedores especializados: sacarose, gelatina bovina Bloom 180 (Gelita do Brasil ltda, Cotia-SP), aroma maçã verde e corante clorofila (Duas Rodas Ind., Jaraguá do Sul-SC), citrato de sódio, ácido cítrico, cloreto de sódio, carbonato de cálcio, ciclamato de sódio, sacarina sódica (Sweetmix Ltda, Sorocaba-SP), inulina e frutooligossacarideos (Clariant S/A, São Paulo-SP), polidextrose (Tovani, São Paulo-SP).

Foram desenvolvidas sete formulações de gelatina funcional contendo diferentes tipos e proporções de fibra alimentar (INU, FOS e PD), conforme tabela 1, com o intuito de avaliar o efeito nas propriedades sensoriais das sobremesas de gelatina. A fórmula padrão contendo sacarose foi desenvolvida com a finalidade de se obter um produto de referência, que apresentasse as propriedades coloidais características da sobremesa de gelatina. Os aditivos foram utilizados de acordo com a necessidade tecnológica, conforme normas do Ministério da Saúde (BRASIL, 1999).

As diferentes formulações de gelatina foram preparadas com a dissolução do pó de cada composição em $250 \mathrm{ml}$ de água quente $\left(100^{\circ} \mathrm{C}\right)$, seguido da adição de $250 \mathrm{~mL}$ de água fria $\left(25^{\circ} \mathrm{C}\right)$. Após completa mistura, triplicatas de $150 \mathrm{~mL}$ de cada formulação foram acondicionadas em béquer de vidro e submetidas à refrigeração até a geleificação.

Para determinar a textura instrumental, quatro parâmetros foram analisados: firmeza, consistência, coesividade e índice de viscosidade. Foi utilizado o analisador de textura (texturômetro) TA-XT2 (Stable Micro Systems, Inglaterra), com célula de carga de $50 \mathrm{~kg}$, sensor ou probe cilíndrico. A velocidade de penetração do probe foi de $0,83 \mathrm{~mm} \mathrm{~s}^{-1}$, a distância de penetração foi de $11 \mathrm{~mm}$ e a velocidade de retorno do sensor foi de $10 \mathrm{~mm} \mathrm{~s}^{-1}$. O probe utilizado foi o modelo esférico $(\mathrm{P} /$ 5S) $20 \mathrm{~mm}$ de diâmetro. Os corpos de prova apresentavam $6,0 \mathrm{~cm}$ de diâmetro por $8,0 \mathrm{~cm}$ de altura e 
Tabela 1 - Formulações padrão e funcionais para sobremesa de gelatina.

\begin{tabular}{|c|c|c|c|c|c|c|c|c|}
\hline \multirow{2}{*}{ Ingredientes (g) } & \multicolumn{8}{|c|}{ 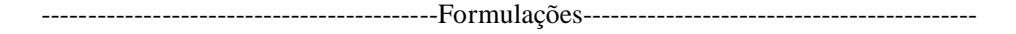 } \\
\hline & Padrão & F1 & $\mathrm{F} 2$ & F3 & F4 & F5 & F6 & F7 \\
\hline Sacarose & 75 & --- & --- & --- & --- & --- & --- & --- \\
\hline Gelatina bovina Bloom 180 & 15 & 15 & 15 & 15 & 15 & 15 & 15 & 15 \\
\hline Frutooligossacarideos (FOS) & --- & 15 & --- & --- & 7,50 & 7,50 & --- & 5 \\
\hline Inulina (INU) & --- & --- & 15 & --- & 7,50 & --- & 7,50 & 5 \\
\hline Polidextrose (PD) & --- & --- & --- & 15 & --- & 7,50 & 7,50 & 5 \\
\hline Extrato de erva-mate verde & 4,50 & 4,50 & 4,50 & 4,50 & 4,50 & 4,50 & 4,50 & 4,50 \\
\hline Aroma natural de maçã verde & 0,97 & 0,97 & 0,97 & 0,97 & 0,97 & 0,97 & 0,97 & 0,97 \\
\hline Citrato de sódio (A) & 0,94 & 0,94 & 0,94 & 0,94 & 0,94 & 0,94 & 0,94 & 0,94 \\
\hline Ácido cítrico (B) & 0,32 & 0,32 & 0,32 & 0,32 & 0,32 & 0,32 & 0,32 & 0,32 \\
\hline Cloreto de sódio & 0,20 & 0,20 & 0,20 & 0,20 & 0,20 & 0,20 & 0,20 & 0,20 \\
\hline Carbonato de cálcio (C) & 0,05 & 0,05 & 0,05 & 0,05 & 0,05 & 0,05 & 0,05 & 0,05 \\
\hline Corante clorofila & 0,02 & 0,02 & 0,02 & 0,02 & 0,02 & 0,02 & 0,02 & 0,02 \\
\hline Ciclamato de sódio (D) & --- & 0,20 & 0,20 & 0,20 & 0,20 & 0,20 & 0,20 & 0,20 \\
\hline Sacarina sódica (D) & --- & 0,07 & 0,07 & 0,07 & 0,07 & 0,07 & 0,07 & 0,07 \\
\hline Massa Total * & 97 & 37,27 & 37,27 & 37,27 & 37,27 & 37,27 & 37,27 & 37,27 \\
\hline
\end{tabular}

$\mathrm{A}=$ regulador de acidez $\mathrm{B}=$ acidulante $\mathrm{C}=$ antiumectante $\mathrm{D}=$ edulcorante

* Massa total para ser adicionado em $500 \mathrm{ml}$ de água.

as leituras foram realizadas em triplicata à temperatura ambiente.

A análise sensorial foi realizada com as formulações de gelatina funcional (com fibras) que não diferem significativamente $(\mathrm{P}>0,05)$ da fórmula padrão quanto à textura instrumental. A análise sensorial foi realizada por uma equipe de 36 provadores de ambos os sexos, não treinados, na faixa etária de 23 a 52 anos, instruídos para a participação. As sobremesas foram codificadas com números ao acaso e oferecidas de forma aleatória aos provadores (FERREIRA et al., 2000). Para avaliar os atributos de sabor e preferência de compra, foi aplicado o teste de comparação múltipla ou diferença-do-controle com uma escala hedônica de nove pontos (ABNT, 1995).

O teor dos compostos fenólicos (ácido cafeíco, 5-CQA e rutina) no extrato de erva-mate em pó foi determinado em sistema de cromatografia a líquido de alta eficiência (CLAE), segundo procedimento estabelecido por DUTRA et al. (2010). O teor de cafeína no extrato de erva-mate em pó também foi determinado em (CLAE) segundo procedimento descrito por DUTRA (2008). A quantificaçãona amostra foi realizada por padronização externa. Todas as determinações na amostra foram conduzidas em triplicata. Os padrões de ácido cafeíco, 5-CQA, rutina e cafeína, todos reagentes Sigma.

As análises de proteína, gordura total e fibra alimentar foram determinadas nas formulações funcionais selecionadas e na fórmula padrão, de acordo com as metodologias oficiais da AOAC INTERNATIONAL (2000) e as análises de umidade e cinzas foram realizadas conforme métodos do INSTITUTOADOLFOLUTZ(2005).

A análise estatística dos resultados foi processada por análise de variância (ANOVA) e teste de Tukey, adotando um valor de $\alpha$ de 0,05 (5\%) (MSTATC, 1989; BARROS NETO et al., 2002).

\section{RESULTADOS E DISCUSSÃO}

Os atributos de firmeza e coesividade são os parâmetros de textura instrumental de maior importância para caracterização das formulações de gelatina testadas neste experimento e corroboram as hipóteses descritas por BOURNE (1982). A medida de resistência à penetração do probe (analisador de textura) como índice de firmeza de géis e produtos gelificados é um ensaio muito comum para qualificar a textura. Nesse mesmo sentido, pode-se citar a coesividade como a força contrária à penetração, caracterizando assim o grau de gomosidade das amostras.

Para os resultados obtidos nesse estudo, pode ser descrita uma relação diretamente proporcional para as formulações testadas, pois quanto maior a firmeza da amostra maior foi sua força de gel e consequentemente maior a sua coesividade (Tabela 2). A formulação (F6) com a mistura INU/PD apresentou valores para firmeza e coesividade que foram 
Tabela 2 - Propriedades coloidais para formulações de gelatina pronta para consumo.

\begin{tabular}{|c|c|c|c|c|}
\hline \multirow{2}{*}{ Formulações } & \multicolumn{4}{|c|}{ - } \\
\hline & Firmeza (g) & Consistência (gs) & Coesividade (gs) & Viscosidade (gs) \\
\hline Padrão & $501,56 \pm 62,29^{\mathrm{bc}}$ & $8377,62 \pm 663,45^{\mathrm{ab}}$ & $35,83 \pm 5,86^{\text {bc }}$ & $362,43 \pm 110,31^{\mathrm{ab}}$ \\
\hline Fórmula F1 & $162,04 \pm 20,29^{\mathrm{d}}$ & $2857,53 \pm 157,86^{\mathrm{c}}$ & $21,12 \pm 1,13^{\mathrm{d}}$ & $267,44 \pm 27,34^{\mathrm{b}}$ \\
\hline Fórmula F2 & $576,37 \pm 41,36^{\mathrm{b}}$ & $9076,58 \pm 1278,19^{\mathrm{ab}}$ & $44,87 \pm 3,97^{\mathrm{b}}$ & $453,44 \pm 122,12^{\mathrm{ab}}$ \\
\hline Fórmula F3 & $586,39 \pm 37,36^{\mathrm{ab}}$ & $8631,78 \pm 656,13^{a b}$ & $39,02 \pm 0,89^{\mathrm{bc}}$ & $321,46 \pm 92,93^{\mathrm{b}}$ \\
\hline Fórmula F4 & $149,14 \pm 10,74^{\mathrm{d}}$ & $2823,02 \pm 316,17^{\mathrm{c}}$ & $21,63 \pm 3,06^{\mathrm{d}}$ & $304,56 \pm 39,71^{\mathrm{b}}$ \\
\hline Fórmula F5 & $193,93 \pm 19,87^{\mathrm{d}}$ & $3602,22 \pm 115,41^{\mathrm{c}}$ & $21,00 \pm 1,97^{\mathrm{d}}$ & $244,17 \pm 75,77^{\mathrm{b}}$ \\
\hline Fórmula F6 & $686,65 \pm 40,06^{\mathrm{a}}$ & $10687,29 \pm 1802,81^{\mathrm{a}}$ & $68,83 \pm 5,79^{\mathrm{a}}$ & $595,12 \pm 164,06^{\mathrm{a}}$ \\
\hline Fórmula F7 & $460,50 \pm 43,79^{c}$ & $7554,60 \pm 1397,50^{\mathrm{b}}$ & $31,86 \pm 5,51^{\mathrm{cd}}$ & $325,41 \pm 49,97^{\mathrm{ab}}$ \\
\hline
\end{tabular}

Nota: FOS = Frutooligossacarídeo; INU= Inulina; $\mathrm{PD}=$ Polidextrose

Médias com letras iguais na mesma coluna não diferem entre si $(\mathrm{P}>0.05)$.

significativamente diferentes $(\mathrm{P}<0.05)$ e superiores ao padrão, $16 \%$ e $94 \%$ respectivamente. Os parâmetros de textura para essa formulação não foram considerados como habituais e desejados para uma sobremesa de gelatina.

As formulações contendo FOS (F1) e FOS adicionada de inulina (F4) e polidextrose (F5) apresentaram atributos de textura similares. A firmeza e a coesividade foram significativamente $(\mathrm{P}<0.05)$ menores com relação à formulação padrão, exceto para o índice de viscosidade (Tabela 2). Entretanto, a textura instrumental revelou que o FOS, em menor concentração e combinado com a inulina e polidextrose (FOS/INU/PD), conferiu à formulação (F7) uma textura que não difere $(\mathrm{P}>0.05)$ do padrão com sacarose, assim como as formulações com simples adição de inulina (F2) e polidextrose (F3) que também não apresentam diferença significativa $(\mathrm{P}>0.05)$. Dessa forma, as formulações INU, PD e FOS/INU/PD foram preparadas para análise sensorial e também foram analisadas quanto à composição química.

Os resultados do teste de diferença-docontrole e preferência de sabor estão expressos na tabela 3 , permitindo verificar que as formulações com adição de inulina ou polidextrose apresentaram diferença significativa $(\mathrm{P}<0,05)$ da fórmula padrão e entre si, com relação ao sabor. Somente a composição (FOS/INU/ $\mathrm{PD})$ não apresentou diferença do padrão $(\mathrm{P}>0.05)$ quanto ao atributo sabor. Os resultados obtidos confirmam que a formulação com simples adição de inulina obteve maior frequência do índice hedônico, que se refere na escala como "melhor que o padrão" com $71 \%$ de preferência de compra. A funcionalidade tecnológica da inulina está baseada no seu efeito em soluções aquosas, que, em determinadas concentrações, causa aumento da viscosidade e pode ser utilizada como um modificador reológico. A sobremesa contendo polidextrose (PD) foi considerada pelo grupo de provadores como a segunda opção na preferência de sabor, porém com preferência de compra em torno de $50 \%$ menor que a primeira opção.

Dentre as fórmulas analisadas sensorialmente, a gelatina padrão e a composição (FOS/ INU/PD) apresentaram as menores médias de aceitação de sabor e preferência de compra. O estudo evidenciou que o atributo sabor de um alimento pode ser modificado pelo tipo de fibra alimentar presente na formulação, mesmo que essa fibra alimentar não atribua gosto ao produto final. Dessa forma, a mistura entre fibras, preferencialmente a inulina, e outros ingredientes como o extrato de erva-mate e aromatizantes conferiu um sabor característico e agradável ao alimento formulado.

As formulações com fibras apresentaram redução de até $92 \%$ no conteúdo de açúcares e consequentemente uma redução média de $77,53 \%$ no valor energético, devido à substituição da sacarose por fibras solúveis. Nas formulações avaliadas, não

Tabela 3 - Teste de diferença-do-controle das fórmulas de gelatina e preferência de sabor.

\begin{tabular}{lcc}
\hline Amostras & Médias \pm DP & $\begin{array}{c}\text { Preferência de } \\
\text { compra }\end{array}$ \\
\hline Fórmula padrão (sacarose) & $3,53 \pm 1,62^{\mathrm{a}}$ & $6,0 \%$ \\
Fórmula F2 (INU) & $6,50 \pm 1,57^{\mathrm{b}}$ & $71,0 \%$ \\
Fórmula F3 (PD) & $4,83 \pm 3,91^{\mathrm{c}}$ & $17,0 \%$ \\
Fórmula F7 (FOS/INU/PD) & $3,64 \pm 2,75^{\mathrm{a}}$ & $6,0 \%$ \\
\hline
\end{tabular}

Médias com letras iguais na mesma coluna não diferem entre si $(\mathrm{P}>0.05)$.

FOS= Frutooligossacarídeo; INU= Inulina; PD= Polidextrose . 
foram observadas diferenças significativas $(\mathrm{P}>0,05)$ nos teores de proteínas e gorduras (Tabela 4). A adição de fibras solúveis à gelatina funcional permitiu que a sobremesa pudesse ser considerada fonte de fibra alimentar, atendendo o regulamento técnico para informação nutricional complementar (BRASIL, 1998b). A quantidade de $3 \%$ de fibra alimentar no produto pronto para consumo é o requisito mínimo para declaração de alegação funcional na rotulagem, como auxiliar no funcionamento do intestino ou contribuindo para o equilíbrio da flora intestinal.

O conteúdo dos compostos bioativos (ácido 5-cafeilquínico, rutina, ácido cafeíco e cafeína) não apresentou diferença significativa $(\mathrm{P}>0,05)$ entre as formulações analisadas (Tabela 4). Para o extrato de erva-mate utilizado, o somatório de compostos fenólicos quantificados foi um valor médio de $88,52 \mathrm{mg}$ para 100g de sobremesa de gelatina preparada. Os compostos fenólicos são os antioxidantes mais abundantes da dieta. O consumo diário pode atingir $1 \mathrm{~g}$ por dia em uma dieta que inclua frutas, hortaliças, chá e vinho tinto. Apesar da distribuição abundante em plantas, os efeitos desses compostos na saúde humana tornaram-se foco de atenção apenas na década de 90 (MANACH et al., 2004). As ações fisiológicas exercidas pelos compostos fenólicos estão relacionadas à redução do risco de doenças em função da elevada capacidade antioxidante (SCALBERT et al., 2005). Quanto mais os antioxidantes sequestram os radicais livres, menor a chance de o indivíduo desenvolver diversas doenças crônico-degenerativas (STORZ, 2006).
A ingestão diária de cafeína deve ser moderada, com doses de até $300 \mathrm{mg}$ para adultos. Com base nos dados de consumo de produtos contendo cafeína, pode-se recomendar a ingestão diária de $4 \mathrm{mg}$ $\mathrm{kg}^{-1}$ para adultos e $1 \mathrm{mg} \mathrm{kg}^{-1}$ para crianças e jovens até 18 anos (BARONE \& ROBERTS, 1996). O consumo de $100 \mathrm{~g}$ de gelatina funcional de erva-mate contribui com a ingestão média de 16,70mg de cafeína, que equivale a $5,6 \%$ da dose recomendada para adultos. Portanto, as formulações podem ser consideradas seguras quanto à ingestão desse alcalóide.

\section{CONCLUSÃO}

A inulina adicionada à gelatina funcional apresentou um índice satisfatório de aceitação, pois realçou o sabor e aumentou a preferência de compra dos provadores. Além disso, as formulações elaboradas com inulina (F2), polidextrose (F3) e a mistura FOS/ INU/PD (F7) apresentaram propriedades coloidais similares à gelatina padrão com sacarose.

As formulações desenvolvidas com fibras e extrato de erva-mate apresentaram como diferencial o baixo valor calórico. Também apresentaram os atributos fonte de fibras e substâncias bioativas da erva-mate, além de ser um alimento dietético. Dessa forma, a gelatina funcional e dietética pode ser consumida por diabéticos e por consumidores que fazem uso de alimentos funcionais.

Tabela 4 - Composição química para sobremesas de gelatina.

\begin{tabular}{|c|c|c|c|c|}
\hline \multirow{2}{*}{ Componentes } & \multirow[b]{2}{*}{ Padrão } & \multirow[b]{2}{*}{ F2 (INU) } & \multirow[b]{2}{*}{ F3 (PD) } & \multirow[b]{2}{*}{ F7 (FOS/INU/PD) } \\
\hline & & & & \\
\hline Carboidratos (g) & $19,10^{\mathrm{a}}$ & $1,51 \mathrm{~b}$ & $1,42 b$ & $1,64 b$ \\
\hline Proteínas (g) & $3,16 \mathrm{a}$ & $3,27 \mathrm{a}$ & $3,18 \mathrm{a}$ & $3,20 \mathrm{a}$ \\
\hline Gorduras totais $(\mathrm{g})$ & $0,03 \mathrm{a}$ & $0,02 \mathrm{a}$ & $0,09 \mathrm{a}$ & $0,04 \mathrm{a}$ \\
\hline Fibras alimentares (g) & $0,00 \mathrm{~b}$ & $3,22 \mathrm{a}$ & $3,45 \mathrm{a}$ & $3,36 \mathrm{a}$ \\
\hline Água (g) & $77,48 b$ & $91,74 \mathrm{a}$ & $91,59 \mathrm{a}$ & $91,51 \mathrm{a}$ \\
\hline Cinzas $(\mathrm{g})$ & $0,12 \mathrm{a}$ & $0,13 \mathrm{a}$ & $0,16 \mathrm{a}$ & $0,14 \mathrm{a}$ \\
\hline Ácido 5-cafeilquínico (mg) & $82,26 \mathrm{a}$ & $82,36 \mathrm{a}$ & $82,32 \mathrm{a}$ & $82,24 a$ \\
\hline Rutina (mg) & $4,84 \mathrm{a}$ & $4,82 \mathrm{a}$ & $4,77 \mathrm{a}$ & $4,93 \mathrm{a}$ \\
\hline Ácido cafeíco (mg) & $1,39 \mathrm{a}$ & $1,46 \mathrm{a}$ & $1,43 \mathrm{a}$ & $1,28 \mathrm{a}$ \\
\hline Cafeína (mg) & $16,69 \mathrm{a}$ & $16,38 \mathrm{a}$ & $16,79 \mathrm{a}$ & $16,91 \mathrm{a}$ \\
\hline Valor energético, kcal/100g & $89,3 \mathrm{a}$ & $19,3 b$ & $19,2 b$ & $19,7 \mathrm{~b}$ \\
\hline
\end{tabular}

Médias com letras iguais na mesma linha não diferem entre si ( $\mathrm{P}>0.05)$. FOS= Frutooligossacarídeo; INU= Inulina; $\mathrm{PD}=$ Polidextrose . 


\section{REFERÊNCIAS}

ABNT. Associação Brasileira de Normas Técnicas. Teste de comparação múltipla em análise sensorial de alimentos e bebidas: NBR 13.526. Rio de Janeiro, 1995. 9p.

AOAC. ASSOCIATION OF OFFICIAL ANALYTICAL CHEMISTS. Official methods of analysis of AOAC International. 17.ed. Gaithersburg, 2000. 2v.

BARONE, J.J.; ROBERTS, H. Caffeine consumption. Food and Chemical Toxicology, v.34, p.119-129, 1996. Disponível em: $<\mathrm{h} \mathrm{t} \mathrm{t} \mathrm{p} \mathrm{:} \mathrm{//} \mathrm{w} \mathrm{w} \mathrm{w.} \mathrm{s} \mathrm{c} \mathrm{i} \mathrm{e} \mathrm{n} \mathrm{c} \mathrm{e} \mathrm{direct.} \mathrm{co} \mathrm{m/}$ science?_ob=ArticleURL\&_udi=B6T6P-3VXBNBT-D\& _user $=10 \&$ rdoc $=1 \&$ fmt=high $\&$ orig $=$ rslt_list \&_origin=rsit_list \&

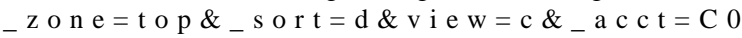
$00050221 \&$ \& version $=1 \&$ \&urlVersion $=0 \&$ \& userid $=10 \&$ md5 $=9 \mathrm{adc} 498 \mathrm{c} 4 \mathrm{bbd} 375 \mathrm{f} 71643 \mathrm{eeb} 23 \mathrm{~d} 4225 \mathrm{c} \&$ searchtype $=\mathrm{a}>$. Acesso em: 17 dez. 2010. doi:10.1016/0278-6915(95)00093-3.

BARROS NETO, B. et al. Como fazer experimentos: pesquisa e desenvolvimento na ciência e na indústria. 2.ed. Campinas: Unicamp, 2002. 401p.

BRASIL. Ministério da Saúde. Portaria n.29, de 13 de janeiro de 1998a. Aprova o Regulamento técnico referente a alimentos para fins especiais. Diário Oficial [da] Republica Federativa do Brasil, Brasília, DF, 20 de março de 1998. Seção 1 .

BRASIL. Ministério da Saúde. Portaria n.27, de 13 de janeiro de 1998b. Regulamento técnico referente à declaração de informação nutricional complementar. Diário Oficial [da] República Federativa do Brasil, Brasília, DF, 30 de março de 1998. Seção 1.

BRASIL. Ministério da Saúde. Resolução n.386, de 05 de agosto de 1999. Regulamento técnico de aditivos utilizados segundo as boas práticas de fabricação e suas funções. Diário Oficial [da] Republica Federativa do Brasil, Brasília, DF, 09 de agosto de 1999. Seção 1.

BRASIL. Ministério de Saúde. Resolução RDC n.02, de 07 de janeiro de 2002. Regulamento Técnico de Substâncias Bioativas e Probióticos Isolados com Alegação de Propriedades Funcionais e ou de Saúde. Diário Oficial [da] República Federativa do Brasil, Brasília, DF, 09 de janeiro de 2002. Seção 1.

BOURNE, M.C. Food texture and viscosity: concept and measurement. New York: Academic, 1982. 325p.

BURGARDT, A.C. Desenvolvimento de uma bebida, utilizando extrato de erva-mate verde (Ilex paraguariensis). 2000. 113f. Dissertação (Mestrado em Tecnologia de Alimentos) - Universidade Federal do Paraná, Curitiba, PR.

CARABIN, I.G.; FLAMM, W.G. Evaluation of safety of inulin and oligofructose as dietary fiber. Regulatory Toxicology and Pharmacology, v.30, p.268-282, 1999. Disponível em: $<\mathrm{h} \mathrm{t} \mathrm{p} \mathrm{:} \mathrm{/} \mathrm{/} \mathrm{w} \mathrm{w} \mathrm{w.} \mathrm{s} \mathrm{c} \mathrm{i} \mathrm{e} \mathrm{n} \mathrm{c} \mathrm{ed} \mathrm{i} \mathrm{r} \mathrm{e} \mathrm{c} \mathrm{t.} \mathrm{c} \mathrm{o} \mathrm{m/}$ science?_ob=ArticleURL\&_udi=B6WPT-45FJXYN-C\&_user $=10$ \&_coverDate $=12 \% 2 \mathrm{~F} 31 \% 2 \mathrm{~F} 1999$ \&_alid $=1582346069$ \&_rdoc $=1 \&$ fmt $=$ high \&_orig $=$ search \&_origin $=$ search\&_zone=rslt_lis t_item\&_cdi=6999\&_sort $=r \& \_s t=13 \&$ docanchor $=\& v i e w=c \&$ _ct $=1 \&$ \& acct $=$ C 000050221 \&_version $=1 \& \_u r l$ Version $=0$ \&_use rid=10\&md5=2e443ddc308643103359f980c801d96b\&searchtype $=a$ > . Acesso em: 17 dez. 2010. doi:10.1006/rtph.1999.1349.
CLIFFORD, M.N. Diet-derived phenols in plasma and tissues and their implications for health. Planta Medica, v.70, p.1103-1114, 2004. Disponível em: <https://www.thiemeconnect.de/ejournals/html/plantamedica/doi/10.1055/s-2004835835>. Acesso em: $17 \mathrm{dez}$. 2010. doi: 10.1055/s-2004835835 .

DUTRA, F.L.G. Compostos fenólicos e metilxantinas em erva-mate armazenada em sistemas de estacionamento natural e acelerado. 2008. 73f. Dissertação (Mestrado em Tecnologia de Alimentos) - Universidade Federal do Paraná, Curitiba, PR.

DUTRA, F.L.G. et al. Determinação de compostos fenólicos por cromatografia líquida de alta eficiência isocrática durante estacionamento da erva-mate. Química Nova, v.33, p.119123, 2010. Disponível em: <http://www.scielo.br/ scielo.php? script=sci_arttext\&pid=S $0100-40$ $422010000100022 \& \operatorname{lng}=e n \& n r m=i s o \& t \operatorname{lng}=p t>$. Acesso em: 17 dez. 2010. doi: 10.1590/S0100-40422010000100022.

FERREIRA, V.L.P. et al. Análise sensorial: testes discriminativos e afetivos. Campinas: SBCTA, 2000. 127p.

FILIP, R. et al. Antioxidant activity of Ilex paraguariensis and related species. Nutrition Research, v.20, p.1437-1446, 2000. Disponível em: <http://www.sciencedirect.com/science?_ob=ArticleURL\&_udi=B6TB145 Y 4 G6S-7\&_user $=10 \&$ _coverDate $=10 \% 2$ F $31 \% 2$ F2000\&_alid $=1582369899 \&$ \&doc $=1 \&$ \& fmt $=$ high \& _orig $=$ search\&_origin $=$ search\&_zone $=$ rslt_list_item\&_cdi $=5129$ \&_sort $=r \&$ st $=13 \&$ _docanchor $=\& v i e w=c \&$ c t $=1 \&$ _acct $=$ C000050221\&_version $=1 \&$ \&urlVersion $=0 \&$ _use rid=10\&md5=f1 a2e0ab036203b20780f656cb7b677e\&searchtype $=a>$. Acesso em: 17 dez. 2010. doi:10.1016/S0271-5317(00)80024-X.

FLOOD, M.T. et al. A review of the clinical toleration studies of polydextrose in food. Food and Chemical Toxicology, v.42, p.1531-1542, 2004. Disponível em: <http://

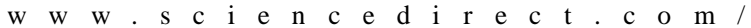

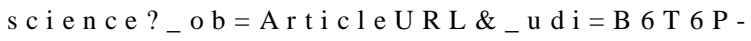
4 CK 7 RFW $-4 \&$ \& user $=10 \&$ _coverDate $=09 \% 2$ F $30 \%$ 2 F2004\&_alid=1582372755\&_rdoc $=1 \&$ fmt $=$ high \&_orig=search\&_origin $=$ search\&_zone $=r$ slt_list_item\&_c $\mathrm{di}=5036 \& \_\mathrm{sort}=\mathrm{r} \& \_\mathrm{st}=13 \& \_d o c a n c h o r=\& v i e w=c \& \_\mathrm{ct}=1 \& \_\mathrm{acct}=\mathrm{C} 000050221$ $\&_{-}$version $=1 \&$ _ur 1 Version $=0 \&$ _userid $=10 \& \mathrm{md} 5$ $=577 \mathrm{~cd} 3 \mathrm{c} 49 \mathrm{ddfffdcaebbc05}$ ae 03 e $6316 \&$ searchtype $=a>$. Acesso em: 17 dez. 2010. doi:10.1016/j.fct.2004.04.015.

IAL (INSTITUTO ADOLFO LUTZ). Normas analíticas: métodos químicos e físicos para análise de alimentos. 5.ed. São Paulo: IAL, 2005. 1v. 533p.

MANACH, C. et al. Polyphenols: food sources and bioavailability. American Journal Clinical Nutrition, v.79, p.727-747, 2004. Disponível em: <http://www.ajen.org/content/ 79/5/727.ful1 ? sid=9a 7 c $9764-84$ d $5-49$ c $1-96$ b 0 9a90a68c0558>. Acesso em: 17 dez. 2010.

MSTATC. Michigan States University. MSTATC versão 2.10. East Lansing, MI, 1989. 2 disquetes 3 1/2 pol., MSDOS.

PARANÁ. Câmara Setorial da Cadeia Produtiva da Erva-Mate. Produtos alternativos e desenvolvimento da tecnologia industrial na cadeia produtiva da erva-mate. Curitiba, 2000. Série PADCT III, n.1. 160p. 
SCALBERT, A. et al. Polyphenols: antioxidants and beyond. American Journal Clinical Nutrition, v.81, p.215-217,

2005. Disponível em: <http://www.ajcn.org/content/81/1/ 215S.full?sid=9ed52a48-d4b0-4662-8ad9-5f0272194b5d>. Acesso em: 17 dez. 2010.

WAITZBERG, D.L. Nutrição oral, enteral e parenteral na prática clínica. 3.ed. Rio de Janeiro: Atheneu, 2000. $534 \mathrm{p}$.
STARK, A.; MADAR, Z. Dietary fiber. In: GOLDBERG, I. Functional foods. New York: Chapman and Hall, 1994. Cap.3, p. 183-201.

STORZ, P. Reactive oxygen species-mediated mitochondriato-nucleus signaling: a key to aging and radical-caused diseases. Science STKE, v.332, p.1-8, 2006. Disponível em: <http:// stke.sciencemag.org/cgi/content/abstract/sigtrans;2006/332/ re3>. Acesso em $17 \mathrm{dez}$. 2010. doi: 10.1126/stke.3322006re3. 